\title{
Temperature-Dependent Size-Controlled Nucleation and Growth of Gold Nanoclusters
}

\author{
Mona B. Mohamed, ${ }^{\dagger}$ Zhong L. Wang,, and Mostafa A. El-Sayed $*, \dagger$ \\ Laser Dynamics Laboratory, School of Chemistry and Biochemistry, Georgia Institute of Technology, \\ Atlanta, Georgia, 30332-0400, and School of Materials Science and Engineering, Georgia Institute of \\ Technology, Atlanta, Georgia, 30332-0245
}

Received: June 15, 1999; In Final Form: August 23, 1999

\begin{abstract}
The electrochemical method of synthesis of gold nanorods in micelles gives substrate solutions that upon spotting and heating on a transmission electron microscope (TEM) substrate result in the nucleation and growth of small gold nanoclusters of narrow size distribution. The size of the nanoclusters, and not their numbers, is found to increased with increasing final temperature to which the substrate is heated. The data are fitted to a mechanism, based on Ostwald ripening in which atomic gold diffusion followed by nucleation on nucleating sites leads to the formation of these small clusters.
\end{abstract}

\section{Introduction}

Great interest in the preparation and characterization of nanostructural materials is driven by many fundamental and practical reasons. ${ }^{1}$ Nanoclusters on the nanometer length scale have been predicted to have unusual electronic, optical, and magnetic properties that lead to technological advanced materials, such as quantum dots in the miniaturization of electronic devices. ${ }^{1,2}$ In addition, these particles have novel catalytic properties and are applied as catalysts and as electrocatalysts. ${ }^{1-7}$ The synthesis of these nanoparticles in solution required a source of metal ions dissolved in an appropriate solvent that were then reduced to the zero valence state. The lifetime of the atoms is short in solution; they tend to coalesce quickly into clusters. The critical step during the preparation of nanoparticles of specific size is carried out by controlling the growth at a certain size and preventing agglomeration. ${ }^{8}$ The growth can be inhibited by using capping material (e.g., ligands, ${ }^{4,8}$ polymers, ${ }^{5,9}$ or surfactant). ${ }^{6,7}$ One of the most well-known method for synthesis of gold nanoparticles is the citrate method that was developed by Turkavich et al. ${ }^{10}$ In this method, $\mathrm{HAuCl}_{3}$ was reduced by sodium citrate; then gold nanocrystals of narrow size distribution (20 $\pm 1 \mathrm{~nm}$ ) was produced. The citrate ions were the reducing and the capping agents. Faraday ${ }^{11}$ prepared colloidal gold nanoparticles in a two-phase system by the reduction of aqueous gold salt with phosphorus in carbon disulfide and obtained a ruby-colored aquous solution of dispersed gold particles. Brust and co-workers ${ }^{12}$ reported a single-step synthesis for thiolcapped gold nanoparticles based on the reduction of $\mathrm{AuCl}_{4}{ }^{-}$ ions by sodium borohydride in the presence of alkanethiol in an aqueous/organic two-phase system to produce very small stable thiol-capped gold nanocrystals having a $2 \pm 1 \mathrm{~nm}$ diameter. Pileni and co-workers ${ }^{13-18}$ reported the shape- and size-controlled synthesis of copper nanoparticles in micelles by varying the surfactant concentration. These authors demonstrated how the shape of the particles could be controlled by the shape of the micellar template. They found that the micelles grew upon an increase in the surfactant concentration or upon the addition of salts, alcohols, or cosurfactants and can form rod-shaped micelles.

\footnotetext{
$\dagger$ School of Chemistry and Biochemistry.

$\leftarrow$ School of Materials Science and Engineering.
}

Electrochemical methods ${ }^{19-22}$ were used to produce transition metal nanoparticles whose size can be controlled by adjusting the current density. In this type of synthesis, tetraalkylammonium salts were used as both supporting electrolyte and stabilizer for the cluster formed. The electrochemical method has been modified $^{23}$ to control the shape of the particle. The gold nanorods can be prepared by using the same electrochemical method in the presence of rod-induced shapes (e.g., cosurfactant), and the catalyst helps the growth and elongation of the particle in one direction.

In this article, we found that during the study of the thermal stability of gold nanorods, when the solution of these nanorods capped with micelles that was synthesized by the electrochemical method is spotted on a TEM substrate and heated, small gold clusters that are $\sim 6.0 \mathrm{~nm}$ in size with very narrow distribution are formed. When the temperature is increased, the size and not the number of these clusters increases. The source of these gold clusters is found to be the gold atoms or very small clusters that did not have enough time to grow. A model is presented that based on Ostwald ripening is used to explain the mechanism of growth of the observed nucleating clusters as the temperature is increased.

\section{Experimental Section}

Gold nanorods were prepared by an electrochemical method..$^{23}$ The electrolyte consists of a hydrophilic cationic surfactant, hexadecyltrimethylammonium bromide, and a hydrophobic cationic cosurfactant, tetradecylammonium bromide or tetraoctylammonium bromide. Small amounts of $\mathrm{Ag}^{+}$in the electrolyte solution are necessary to obtain the rods rather than spheres. The electrolysis was carried out under ultrasonication (in order to keep the micelles in solution). An applied electric field of $2 \mathrm{~V}$ and $\sim 5 \mu \mathrm{A}$ were used. The temperature was adjusted to $42{ }^{\circ} \mathrm{C}$. The electrolysis was carried out for $45 \mathrm{~min}$.

Gold nanorod solutions were dispersed and slowly dried on an ultrathin carbon film supported by a copper mesh. This was used for imaging by using a transmission electron microscope (TEM). The in situ TEM was carried out at $200 \mathrm{kV}$ using a Hitachi HF-2000 TEM equipped with a field emission source. The column vacuum in the TEM was $\sim 2 \times 10^{-8}$ Torr. The in situ heating was carried out electrically using a Gatan TEM specimen heating stage. The TEM images were recorded digitally using a CCD camera.

\subsection{1/jp9919720 CCC: $\$ 18.00$ @ 1999 American Chemical Society} Published on Web 10/04/1999 


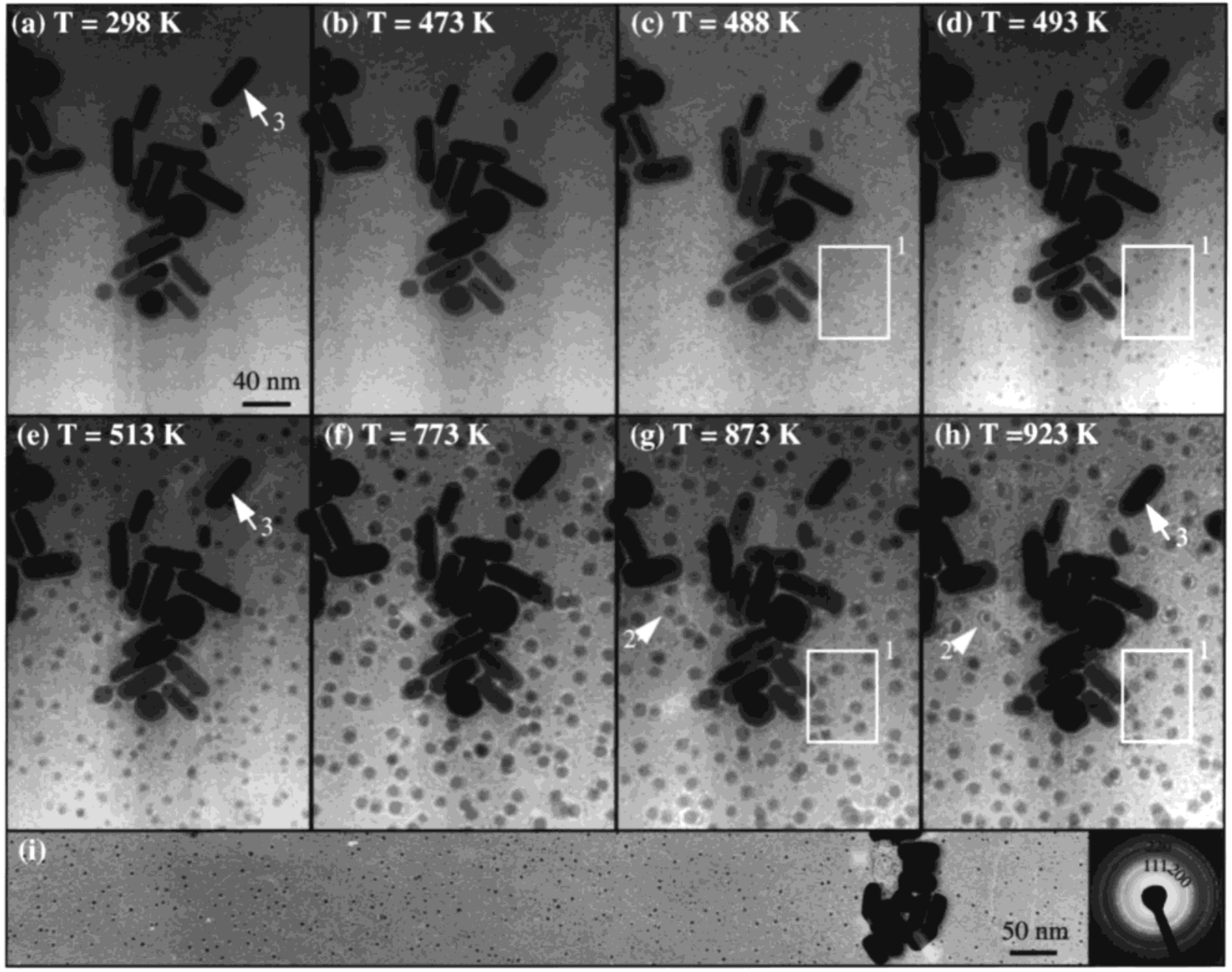

Figure 1. $(\mathrm{a}-\mathrm{h})$ Series of in situ TEM images recorded for the same specimen region as the temperature was increased continuously from room temperature to $923 \mathrm{~K}$, showing the growth in size but not in number of small gold nanoparticles on the carbon support. (i) Low-magnification TEM image recorded from another specimen in a region that was not illuminated by the beam during the in situ experiment to $T=700 \mathrm{~K}$.

\section{Results and Discussion}

The dynamic processes for the formation of these particles were examined in situ by TEM (Figure 1). A series of in situ TEM images recorded for the same specimen region as the temperature was increased continuously from room temperature to $923 \mathrm{~K}$, showing the formation process of Au small nanoparticles on the carbon support in Figure 1. The nucleation started at $473 \mathrm{~K}$, as shown in (Figure 1b), and it was only observed by the TEM at $488 \mathrm{~K}$ (Figure 1c). At $T>513 \mathrm{~K}$, the widths of the nanorods increased significantly while their lengths remained the same (compare the rod marked by an arrow in parts a, e, and h of Figure 1). This is due to the surface melting, which induces shape transformation so that the thickness of the nanorods decreased when placed onto the substrate as a "liquidlike" material (note an increase in the width does not necessarily mean an increase in the volume). Figure 1i shows a low-magnification TEM image recorded from another specimen in a region that was not illuminated by the beam during the in situ experiment to $T=700 \mathrm{~K}$, showing a uniform distribution of the particles on the substrate surface. It is noted that the density of the particles in the regions adjacent to the Au rods is approximately the same as the particles in the regions without rods. This suggests that the origin of the gold atom forming the small clusters is not the gold nanorods. Energydispersive X-ray microanalysis (EDS) indicates that the particles were composed of $\mathrm{Au}$, and the electron diffraction pattern proved that the structure of the particles is face-centered cubic. It must also be pointed out that the beam current used to record the image was rather low in the field-emission TEM and that no significant local heating was introduced for an illumination circle of $\sim 5 \mu \mathrm{m}$ in diameter.

It is clear that as the temperature reached $433 \mathrm{~K}$, at which the structure of the micelles around the rods was found to collapse in solution, ${ }^{24}$ no visible change was observed in the morphology of the nanorods. At $T=488 \mathrm{~K}$ (Figure 1c), small nuclei of gold nanoparticles began to form on the surface of the carbon substrate. A small increase in the temperature to 493 $\mathrm{K}$ (Figure 1d) resulted in a rapid growth of these small particles on the carbon substrate. A further increase in the temperature up to $513 \mathrm{~K}$ led to the further growth of the particles, and the stable particle size was reached at $T=600 \mathrm{~K}$ (Figure 3a). The following phenomena were observed.

(i) The nucleation of the small particles occurred consistently in the $473-488 \mathrm{~K}$ temperature range. This critical temperature range is independent of the substrate used for supporting the gold nanorods, being either carbon or $\mathrm{SiO}_{2}$.

(ii) The density of the nucleation sites did not increase with temperature, although the size of the particles continued to grow (see the area indicated by a rectangular frame in Figure 1).

(iii) For $T>873 \mathrm{~K}$, the sizes of the particles located near the gold nanorods decreased (see the particle indicated by arrowhead 2 in parts $g$ and $\mathrm{h}$ of Figure 1, where the eyeball 

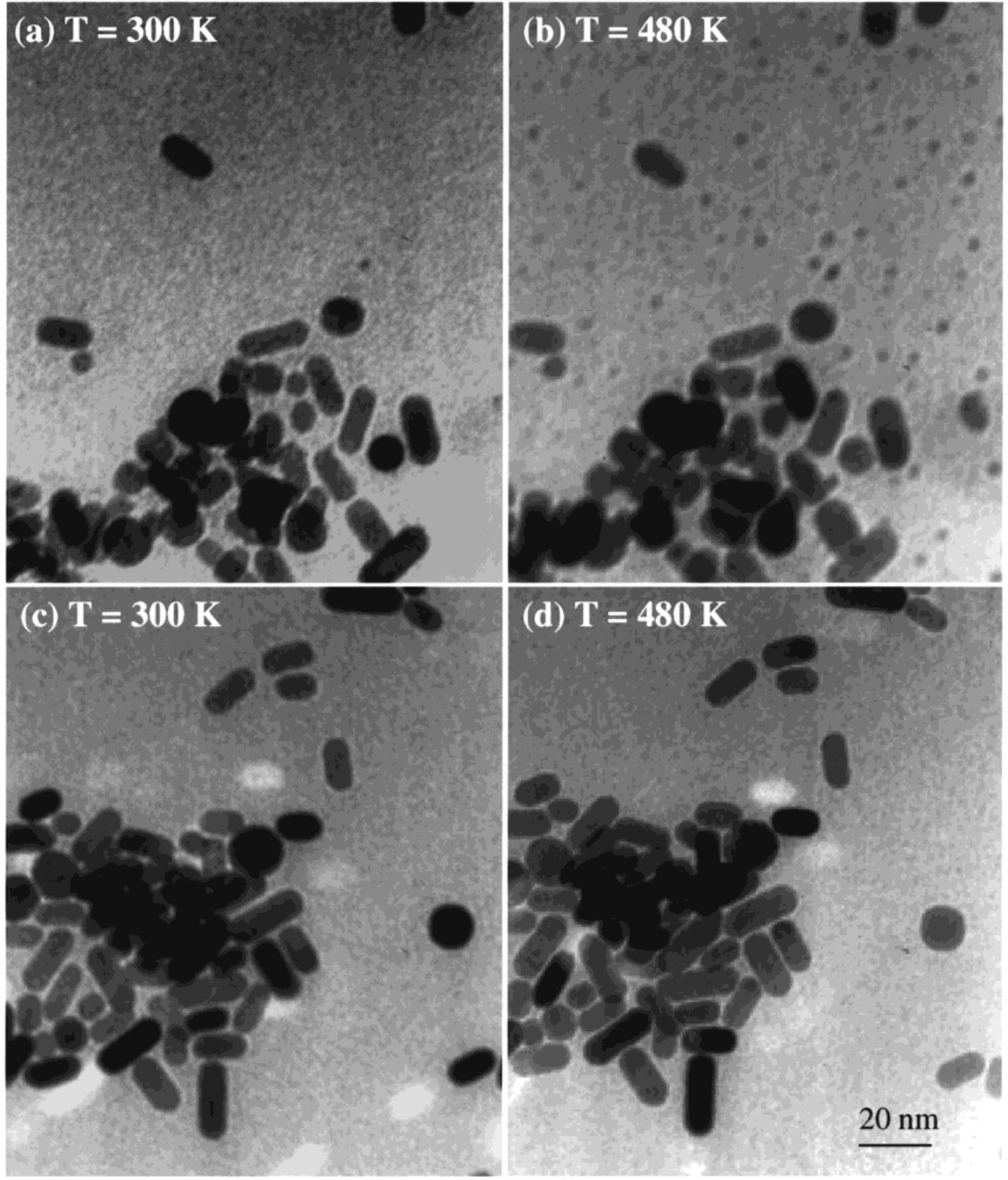

Figure 2. Formation of gold cluster indicated by transmission electron microscopy images recorded in situ: $T=300 \mathrm{~K}$ (a) and $T=480 \mathrm{~K}$ (b) from the as-prepared Au nanorods; with centrifuging and dialysis of the supernatant solution prior to spotting the sample on the substrate and using the top solution (with less surfactant concentration and other gold-containing nanomaterial) (c, d). The formation of small Au particles is observed in the as-prepared nanorod specimen but not in the specimen made from the supernatant solution of centrifuged gold solution.

shape is due to the damage of the particle to the substrate surface), while the size of the particles located further away from the nanorods remained constant (Figure 3a).

(iv) The width of the nanorods increased significantly when the temperature was higher than $873 \mathrm{~K}$, while their lengths remain the same (see the nanorod indicated by arrowhead 3 ). This is due to the partial melting of the nanorods onto the substrate surface and the decrease in the particle thickness. This temperature is much lower than the melting temperature of the Au spheres of diameter $20 \mathrm{~nm}$, which is $1220 \mathrm{~K}$ in the pressure range of the TEM measurements. ${ }^{25}$

(v) The size distribution of the newly formed $\mathrm{Au}$ particles was rather narrow $(6.0 \pm 0.3 \mathrm{~nm})$, and the particles grew uniformly over the entire support (see Figure 1i). The newly formed particles were crystalline, as determined from the electron diffraction pattern shown in the inset of Figure 1i (and from HRTEM images). The growth of Au particles within the beam illumination circle and the regions far from the beam was examined carefully. It was found that the size and distribution of the gold particles were unaffected by the electron beam. ${ }^{26}$ To find out the source of the gold atoms that nucleate and grow into the small nanoclusters, a set of experiments were performed that gave the following observations.

(1) It is found that the as-prepared solution containing spheres and rods gives rise to the formation of in situ TEM small nanoclusters upon heating.

(2) Centrifuging and dialysis of the top solution prior to spotting the sample on the TEM substrate (with less surfactant, gold atoms, or nanomaterials concentration) produced no clusters.

(3) When the bottom component of the centrifuged sample was spotted and heated, it is found to give rise to nucleation 

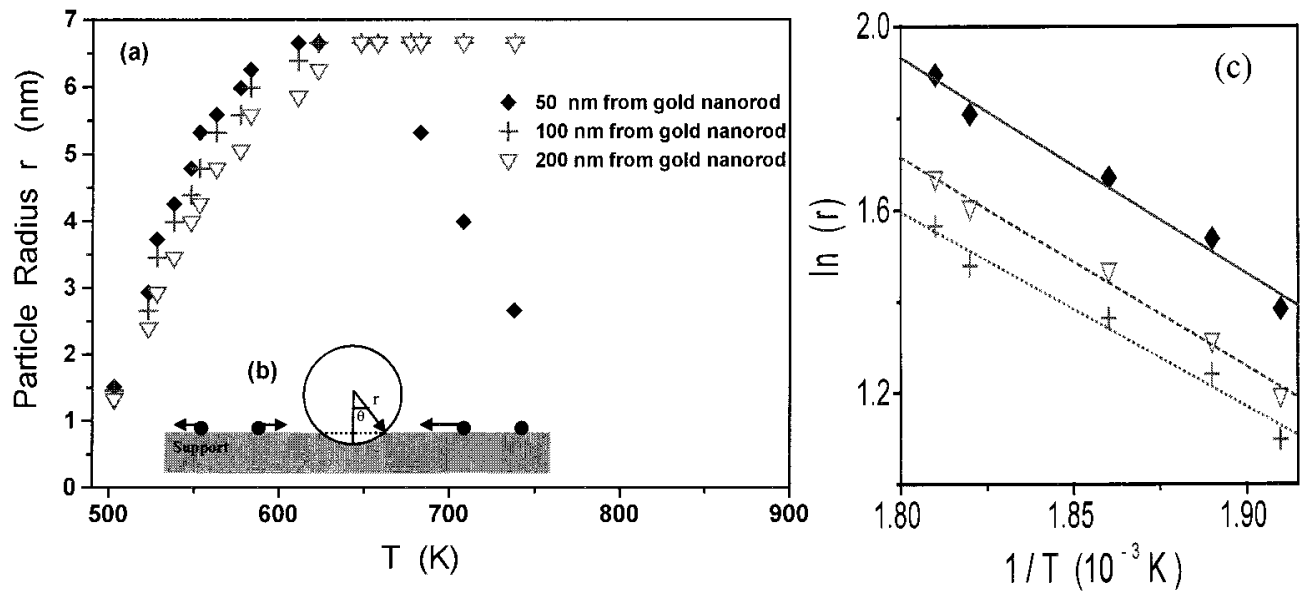

Figure 3. (a) Dependence of the steady-state particle radii $\left(r_{\max }\right)$ formed at different specimen temperatures for particles located at different distances from the Au rods, as represented by different symbols. The size of the particles located closest to the rod ( $\sim 50 \mathrm{~nm})$ dropped sharply at $650 \mathrm{~K}$. Part $\mathrm{b}$ gives a sketch showing the growth process of the small gold cluster on the TEM substrate. Part c shows $\ln \left(r_{\max }\right)$ vs $1 / T$ plot in the low-temperature range, the slopes of which give the difference between the activation energy of diffusion and the sublimation energy $Q$ of the gold atoms.

and growth of the small clusters together with a similar concentration of the rods and regular size of the spheres.

From the above, the source of gold seems to be the small particles that have not completely formed gold rods, or large spheres that have been found to be unstable and to break up in the solution. Most likely, it is from reduced gold ions and very small clusters during the growth to a large rod or sphere but did not have enough time or enough capping material for the complete growth.

\section{Model}

We propose that the formation of small Au nanoparticles in Figure $1 \mathrm{~d}-\mathrm{h}$ is the result of the release of gold atoms from the small gold particles that are below our instrumental resolution followed by surface diffusion on the substrate surface and then nucleation and growth on defect sites. Initially, Au atoms begin to diffuse at a temperature below $493 \mathrm{~K}$ (slightly below this temperature, the unstable micelles around the small particles dissociate). As seen in Figure 1i, the distribution of $\mathrm{Au}$ particles on the carbon support is uniform and monodispersive. The second process is the diffusion of the absorbed gold atoms onto the surface of the substrate. With the increase in the density of surface atoms, nucleation of the gold particles is expected to occur at some defect sites on the amorphous substrate surface.

The initial rapid growth of the Au particles is due to the contribution of the diffusion of atoms on the substrate surface. This process is assumed to follow the hit-and-stick model and is directly related to the intersection length of the particle with the substrate surface. On the other hand, the atoms on the surface of the Au particles can sublime, and their sublimation rate is directly proportional to the exposed surface area of the particle. Accordingly, for a spherical particle of radius $r$, partially embedded in the substrate as shown in Figure 3b, the net growth of the number of atoms $\left(n_{r}\right)$ in the particle is given by

$$
\mathrm{d} n_{r} / \mathrm{d} t=(2 \pi r \sin \theta) N_{0} k_{\mathrm{d}}-4 \pi r^{2} \alpha \rho_{\mathrm{s}} k_{\mathrm{s}}
$$

where $\theta$ is the angle that specifies the degree of contact of the particle with the substrate, $k_{\mathrm{d}}$ is the diffusion rate constant of the atoms adsorbed on the substrate, $N_{0}$ is the steady-state concentration of the $\mathrm{Au}$ atoms on the surface, $\alpha$ is a factor representing the fraction of the exposed surface area, $\rho_{\mathrm{s}}$ is the surface density of the particle, and $k_{\mathrm{s}}$ is the desorption rate constant. The direct condensation of Au atoms onto the particle surface can be accounted for by a proper choice of the $\alpha$ factor. ${ }^{27}$ Initially, the diffusion term is dominant, resulting in a rapid growth of the particle. As the particle grows in size, the desorption term increases because of the $r^{2}$ term. When the two terms are equal, a steady state is reached $\left(\mathrm{d} n_{r} / \mathrm{d} t=0\right)$, achieving a maximum radius $\left(r_{\max }\right)$ at this temperature, which is given by

$$
r_{\max }=\frac{N_{0} k_{\mathrm{d}} \sin \theta}{2 \alpha \rho_{\mathrm{s}} k_{\mathrm{s}}}
$$

By use of the relation of $n_{r}=(4 / 3) \pi r^{3} \rho_{0}$, where $\rho_{0}$ is the volume density of $\mathrm{Au}$ atoms, and the boundary condition of $r=0$ at $t=0$, the solution of eq 1 relating $r$ to $t$ is given by

$$
r_{\max } \ln \left(\frac{r_{\max }}{r_{\max }-r}\right)-r=\left[\frac{\alpha \rho_{s} k_{s}}{\rho_{0}}\right] t
$$

$r_{\max }$ is independent of the location of the particle as long as the local density of surface atoms on the substrate is constant. The kinetics of Au particle growth was determined using in situ TEM at $498 \mathrm{~K}$ as soon as the newly formed particles were observed in the TEM images. From the kinetic curve given in Figure 4a, the particles seems to experience a rapid growth in the first 500 $\mathrm{s}$ and then the size reached a constant value. By setting the left-hand side of eq 3 equal to $Y$, using the experimental data in Figure 4a, a plot of $Y$ vs time gives straight lines (as shown in Figure 4b) with an average slope of $\left[\alpha \rho_{s} k_{s} / \rho_{0}\right]=(3.95 \pm$ $0.1) \times 10^{-3} \mathrm{~nm} / \mathrm{s}$. From this, and using the values for $\rho_{\mathrm{s}}$ and $\rho_{0}$ (28), $\alpha k_{\mathrm{s}}$ is found to be equal to $(1.6 \pm 0.04) \times 10^{-2} \mathrm{~s}^{-1}$ at 498 $\mathrm{K}$. The desorption rate constant, $k_{\mathrm{s}}$ can be approximated by $k_{\mathrm{s}}=v \exp (-Q /(k T)$, where $v$ is the mean $\mathrm{Au}-\mathrm{Au}$ vibration frequency and $Q$ is the desorption activation energy. $v$ is related to the Debye temperature $\left(T_{\mathrm{D}}\right)$ by $v=k T_{\mathrm{D}} / h$, in which $T_{\mathrm{D}}=$ $170 \mathrm{~K} .{ }^{29}$ A calculation for $0.5<\alpha<0.75$ gives $Q=(1.39 \pm$ 0.01 ) eV (note that $Q$ is insensitive to the changes in the value of $\alpha$ used). From eq 2, the steady-state size of the particle $\left(r_{\max }\right)$ is related to the diffusion activation energy (barrier to diffusion) $E_{\mathrm{d}}$ by

$$
r_{\max }=\frac{N_{0} \sin \theta}{2 \alpha \rho_{\mathrm{s}} v} \exp \left(-\frac{E_{0}-Q}{k T}\right)
$$

Plots of $\ln \left(r_{\max }\right)$ vs $1 / T$ (Figure $3 \mathrm{~b}$ ) gave straight lines from 

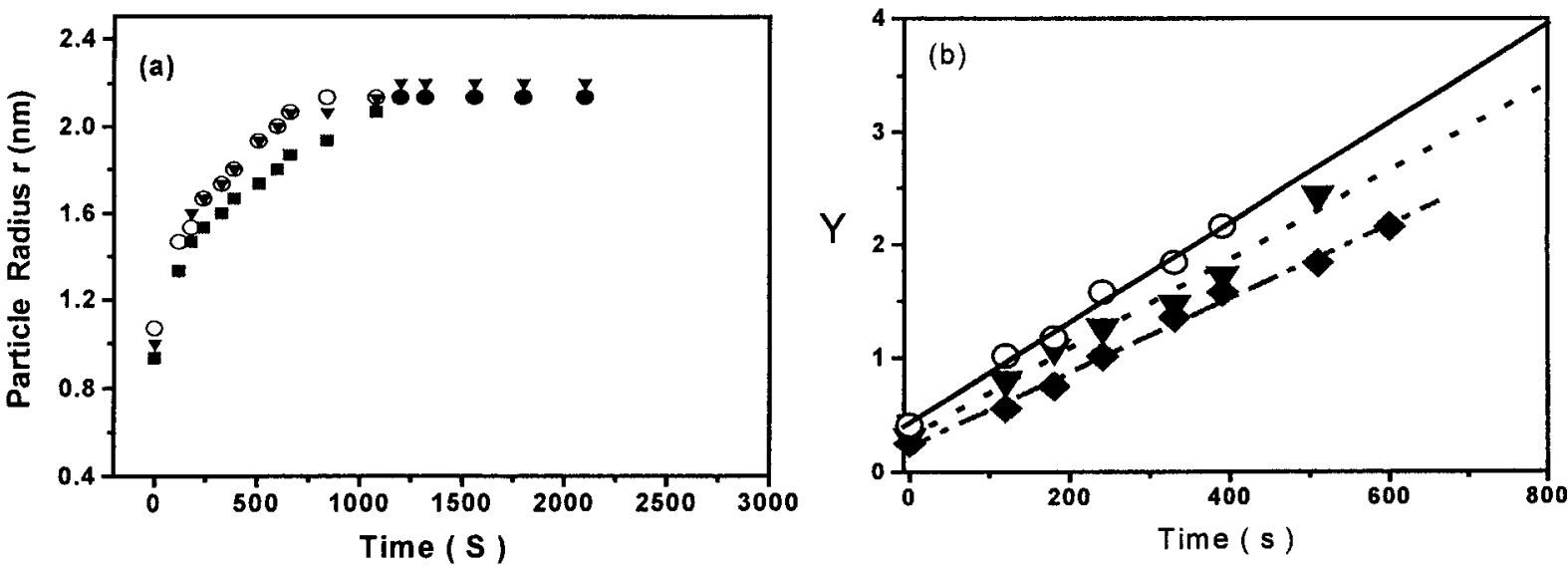

Figure 4. Growth kinetics of the small particles at a constant temperature of $T=498 \mathrm{~K}$ : (a) particle radii as a function of time; (b) comparison of the theoretically calculated $Y$ vs time curves according to eq 3 with the experimentally observed data points. From the slopes of the lines, the sublimation energy $Q$ is calculated.

which $E_{\mathrm{d}}-Q=0.35 \mathrm{eV}$ was calculated. Therefore, the energy barrier to the diffusion of $\mathrm{Au}$ on carbon is $E_{\mathrm{d}}=(1.74 \pm 0.01)$ $\mathrm{eV}$.

As shown previously in Figures $1 \mathrm{~h}$ and $3 \mathrm{a}$, Au particles located near nanorods are found to decrease in size at higher temperatures, while those located at large distances remain unchanged. As the temperature increases, the diffusion rate of the $\mathrm{Au}$ atoms on the substrate surface increases, which results in strong competition between the rods and these particles for the $\mathrm{Au}$ atoms on the carbon surface between them. Because of its larger contact surface area of the rod, more of the Au atoms condense on it. This, together with the tendency for desorption of the gold atoms from the small clusters (with large surface tension) to the rods having larger surface area and small surface tension, leads to the observed decrease in the growth rates of the nearby particles as well as their observed shrinkage at high temperatures.

Acknowledgment. This research was supported by NSF Grants DMR-9733160 and CHE-9727633. We acknowledge Mr. Colin Heyes for the careful reading of the manuscript. Mona Mohamed thanks the Egyptian GM and Molecular Design Institute (MDI) Georgia Institute of Technology for financial support.

\section{References and Notes}

(1) Schmid, G., Ed. Clusters and Colloids; VCH: Weinheim, 1994. Henglin, A. J. Phys. Chem. 1993, 97, 5457.

(2) Weller, H. Angew Chem. 1993, 105, 43. Halperin, W. P. Rev Mod. Phys. 1986, 58, 533.

(3) Schmid, G. Chem Rev. 1992, 92, 1709.

(4) Henglein, A. J. Phys. Chem. 1993, 97, 8457.

(5) Kamat, P. V. Prog. React. Kinet. 1994, 19, 277.

(6) Bradly, J. S.; Millar, J. M.; Rill, E. W. J. Am. Chem. Soc. 1991 113,4016
(7) Toshina, N.; Takahashi, T. Bull. Chem. Soc. Jpn. 1992, 65, 400.

(8) Blum, J.; Sasson, Y.; Zoran, A. J. Mol. Catal. 1981, 11, 293.

(9) Ahmedi, T.; Wang, Z. L.; Green, T. C.; El-Sayed, M. A. Science 1996, 272, 1924

(10) Turkavish, P.; Stevenson, P.; Hillier, J. Faraday Discuss. 1951, 11,55 .

(11) Faraday, M. Philos. Trans. R. Soc. London 1857, 147, 145

(12) Brust, M.; Walker, M.; Bethell, D.; Schiffrin, D. J.; Whyman, R. J. Chem. Soc., Chem. Commun. 1994, 802

(13) Tanori, J.; Pileni, M. P. Langmuir 1997, 13, 639.

(14) Tanori, J.; Pileni, M. P. Adv. Mater. 1995, 7, 862.

(15) Lisieki, I.; Pileni, M. P. J. Am. Chem. Soc. 1993, 115, 3887.

(16) Lisieki, I.; Pileni, M. P. J. Phys. Chem. 1995, 99, 5077. 4160

(18) Lisieki, I.; Borjling, M.; Motte, L.; Ninham, B. W.; Pileni, M. P. Langmuir 1995, 11, 2385.

(19) Reetz, M. T.; Helbig, W. J. Am. Chem. Soc. 1994, 116, 7401

(20) Reetz, M. T.; Helbig, W.; Stimming, U.; Breuer, N.; Vogel, R. Science 1995, 267, 367 .

(21) Reetz, M. T.; Helbig, W.; Quaiser, S. A. Chem. Mater. 1995, 7 , 2227.

(22) Reetz, M. T.; Helbig, W.; Quaiser, S. A. In Active Metals: Preparation, Characterization, and Applications; Furstner, A., Ed.; VCH: Weinheim, 1996.

(23) Yu, Y.; Chang, S.; Lee, C.; Wang, C. R. C. J. Phys. Chem. B 1997, 101 (34), 6661

(24) Mohamed, M. B.; Ismail, K. Z.; Link, S.; El-Sayed, M. A. J. Phys. Chem. B 1998, 102, 9370.

(25) Buffet, Ph.; Borel, J. P. Phys. Rev. A 1976, 13, 2287.

(26) Iijima, S.; Ichihashi, T. Phys. Rev. Lett. 1986, 56, 616. Smith, D J.; Petford-Long, A. K.; Wallenberg, L. R.; Bovin, J.-O. Dyn. Sci. 1986, 233, 872. Ajayan, P. M.; Marks, L. D. Phys. Rev. Lett. 1989, 63, 279.

(27) An adjustment of the $\alpha$ factor can account for the contribution made by the condensation of Au vapor directly onto the particle surface because it is also proportional to the exposed surface area. The fraction of the exposed surface area is related to the angle $\theta$ by $\alpha=(1+\cos \theta) / 2$.

(28) The density of surface atoms is calculated by assuming that $50 \%$ of the surface is $\{111\}$ and $50 \%$ is $\{100\}$ so that the surface density of atoms is $\rho_{\mathrm{s}}=2.333 / a^{2}$, where $a=0.4078 \mathrm{~nm}$, the lattice constant of Au.

(29) Bouesch, P. Phonons: Theory and Experiments I-Lattice Dynamics and Models of Interatomic Forces; Springer-Verlag: Berlin, Heidelberg, New York, 1982. 